\title{
Path-Width and Three-Dimensional Straight-Line Grid Drawings of Graphs *
}

\author{
Vida Dujmović ${ }^{1}$, Pat Morin ${ }^{2}$, and David R. Wood $^{2}$ \\ 1 School of Computer Science, McGill University, Montréal, Canada. \\ vida@cs.mcgill.ca \\ 2 School of Computer Science, Carleton University, Ottawa, Canada. \\ \{morin, davidw\}@scs.carleton.ca
}

\begin{abstract}
We prove that every $n$-vertex graph $G$ with path-width pw $(G)$ has a three-dimensional straight-line grid drawing with $O\left(\operatorname{pw}(G)^{2} \cdot n\right)$ volume. Thus for graphs with bounded path-width the volume is $O(n)$, and it follows that for graphs with bounded tree-width, such as series-parallel graphs, the volume is $O\left(n \log ^{2} n\right)$. No better bound than $O\left(n^{2}\right)$ was previously known for drawings of series-parallel graphs. For planar graphs we obtain three-dimensional drawings with $O\left(n^{2}\right)$ volume and $O(\sqrt{n})$ aspect ratio, whereas all previous constructions with $O\left(n^{2}\right)$ volume have $\Theta(n)$ aspect ratio.
\end{abstract}

\section{Introduction}

The study of straight-line graph drawing in the plane has a long history; see [37] for a recent survey. Motivated by interesting theoretical problems and potential applications in information visualisation [35], VLSI circuit design [26] and software engineering [36], there is a growing body of research in three-dimensional straight-line graph drawing.

Throughout this paper all graphs $G$ are undirected, simple and finite with vertex set $V(G)$ and edge set $E(G) ; n=|V(G)|$ denotes the number of vertices of $G$. A three-dimensional straight-line grid drawing of a graph, henceforth called a three-dimensional drawing, represents the vertices by distinct points in 3 -space with integer coordinates (called grid-points), and represents each edge as a line-segment between its end-vertices, such that edges only intersect at common end-vertices. If a three-dimensional drawing is contained in an axisaligned box with side lengths $X-1, Y-1$ and $Z-1$, then we speak of an $X \times Y \times Z$ three-dimensional drawing with volume $X \cdot Y \cdot Z$ and aspect ratio $\max \{X, Y, Z\} / \min \{X, Y, Z\}$. This paper considers the problem of producing a three-dimensional drawing of a given graph with small volume, and with small aspect ratio as a secondary criterion.

Related Work: In contrast to the case in the plane, every graph has a threedimensional drawing. Such a drawing can be constructed using the 'moment

* Research supported by NSERC and FCAR. 
curve' algorithm in which vertex $v_{i}, 1 \leq i \leq n$, is represented by the grid-point

$$
\left(i, i^{2}, i^{3}\right) \text {. }
$$

It is easily seen - compare with Lemma 4 to follow - that no edges cross. (Two edges cross if they intersect at some point other than a common end-vertex.) Cohen et al. [8] improved the resulting $O\left(n^{6}\right)$ volume bound, by proving that if $p$ is a prime with $n<p \leq 2 n$, and each vertex $v_{i}$ is represented by the grid-point

$$
\left(i, i^{2} \bmod p, i^{3} \bmod p\right)
$$

then there is still no edge crossings. This construction is a generalisation of a twodimensional technique due to Erdös [16]. Furthermore, Cohen et al. [8] proved that the resulting $O\left(n^{3}\right)$ volume bound is asymptotically optimal in the case of the complete graph $K_{n}$, and that every binary tree has a three-dimensional drawing with $O(n \log n)$ volume.

Calamoneri and Sterbini [5] proved that every 4-colourable graph has a threedimensional drawing with $O\left(n^{2}\right)$ volume. Generalising this result, Pach et al. [30] proved that every $k$-colourable graph, for fixed $k \geq 2$, has a three-dimensional drawing with $O\left(n^{2}\right)$ volume, and that this bound is asymptotically optimal for the complete bipartite graph with equal sized bipartitions. If $p$ is a suitably chosen prime, the main step of this algorithm represents the vertices in the $i$ th colour class by grid-points in the set

$$
\left\{(i, t, i t): t \equiv i^{2} \quad(\bmod p)\right\} .
$$

The first linear volume bound was established by Felsner et al. [17], who proved that every outerplanar graph has a drawing with $O(n)$ volume. Their elegant algorithm 'wraps' a two-dimensional layered drawing around a triangular prism; see Lemma 5 for more on this method. Poranen [32] proved that seriesparallel digraphs have upward three-dimensional drawings with $O\left(n^{3}\right)$ volume, and that this bound can be improved to $O\left(n^{2}\right)$ and $O(n)$ in certain special cases. Recently di Giacomo et al. [11] proved that series-parallel graphs with maximum degree three have three-dimensional drawings with linear volume.

Note that three-dimensional drawings with the vertices having real coordinates have been studied by Bruß and Frick [4], Chilakamarri et al. [6], Chrobak et al. [7], Cruz and Twarog [9], Eades and Garvan [15], Garg et al. [18], Hong [22], Hong and Eades [23, 24], Hong et al. [25], Monien et al. [27], and Ostry [29]. Aesthetic criteria besides volume which have been considered include symmetry [22-25], aspect ratio [7, 18], angular resolution [7, 18], edge-separation [7, 18], and convexity $[6,7,15]$.

Tree-Decompositions: Before stating our results we recall some definitions. A tree-decomposition of a graph $G$ is a tree $T$ together with a collection of subsets $T_{x}$ (called bags) of $V(G)$ indexed by the vertices of $T$ such that:

$$
-\bigcup_{x \in V(T)} T_{x}=V(G)
$$


- for every edge $v w \in E(G)$, there is a vertex $x \in V(T)$ such that the bag $T_{x}$ contains both $v$ and $w$, and

- for all vertices $x, y, z \in V(T)$, if $y$ is on the path from $x$ to $z$ in $T$, then $T_{x} \cap T_{z} \subseteq T_{y}$.

The width of a tree-decomposition is the maximum cardinality of a bag minus one. A path-decomposition is a tree-decomposition where the tree $T$ is a path $T=$ $\left(x_{1}, x_{2}, \ldots, x_{m}\right)$, which is simply identified by the sequence of bags $T_{1}, T_{2}, \ldots, T_{m}$ where each $T_{i}=T_{x_{i}}$. The path-width (respectively, tree-width) of a graph $G$, denoted by $\operatorname{pw}(G)(\operatorname{tw}(G))$, is the minimum width of a path-decomposition (treedecomposition) of $G$. A graph $G$ is said to have bounded path-width (tree-width) if $\operatorname{pw}(G)=k(\operatorname{tw}(G)=k)$ for some constant $k$. Given a graph with bounded path-width (tree-width), the algorithm of Bodlaender [1] determines a pathdecomposition (tree-decomposition) with width $\operatorname{pw}(G)(\operatorname{tw}(G))$ in linear time. Note that the relationship between graph drawings and path-width or tree-width has been previously investigated by Dujmović et al. [13], Hliněný [21], and Peng [31], for example.

Our Results: Our main result is the following.

Theorem 1. Every n-vertex graph $G$ has an $O(\operatorname{pw}(G)) \times O(\operatorname{pw}(G)) \times O(n)$ three-dimensional drawing.

Since $\operatorname{pw}(G)<n$, Theorem 1 matches the $O\left(n^{3}\right)$ volume bound discussed above; in fact, the drawings of $K_{n}$ produced by our algorithm are identical to those produced by Cohen et al. [8]. We have the following corollary since every $\operatorname{graph} G$ has $\operatorname{pw}(G) \in O(\operatorname{tw}(G) \cdot \log n)[2]$.

Corollary 1. (a) Every n-vertex graph with bounded path-width has a threedimensional drawing with $O(n)$ volume. (b) Every $n$-vertex graph with bounded tree-width has a three-dimensional drawing with $O\left(n \log ^{2} n\right)$ volume.

While the notion of bounded tree-width may appear to be a purely theoretic construct, graphs arising in many applications of graph drawing do have small tree-width. For example, outerplanar graphs, series-parallel graphs and Halin graphs respectively have tree-width 2,2 and 3 (see $[2,12]$ ). Thus Corollary 1(b) implies that these graphs have three-dimensional drawings with $O\left(n \log ^{2} n\right)$ volume. While linear volume is possible for outerplanar graphs [17], our result is the first known sub-quadratic volume bound for all series-parallel and Halin graphs. Another example arises in software engineering applications. Thorup [34] proved that the control-flow graphs of go-to free programs in many programming languages have tree-width bounded by a small constant; in particular, 3 for Pascal and 6 for C. Other families of graphs having bounded tree-width (for constant $k$ ) include: almost trees with parameter $k$, graphs with a feedback vertex set of size $k$, partial $k$-trees, bandwidth $k$ graphs, cutwidth $k$ graphs, planar graphs of radius $k$, and $k$-outerplanar graphs. If the size of a maximum clique is a constant 
The proofs of Theorems 1 and 2 proceed in three steps. First, an ordered layering with no $\mathrm{X}$-crossing is constructed from a given path-decomposition. The second step balances the number of vertices on each layer. The third step, which is essentially the converse of Lemma 1, takes an ordered layering with no $\mathrm{X}$-crossing and assigns coordinates to the vertices to avoid edge crossings. The style of three-dimensional drawing produced by our algorithm, where vertices on a single layer are positioned on vertical 'rods', is illustrated in Fig. 1.

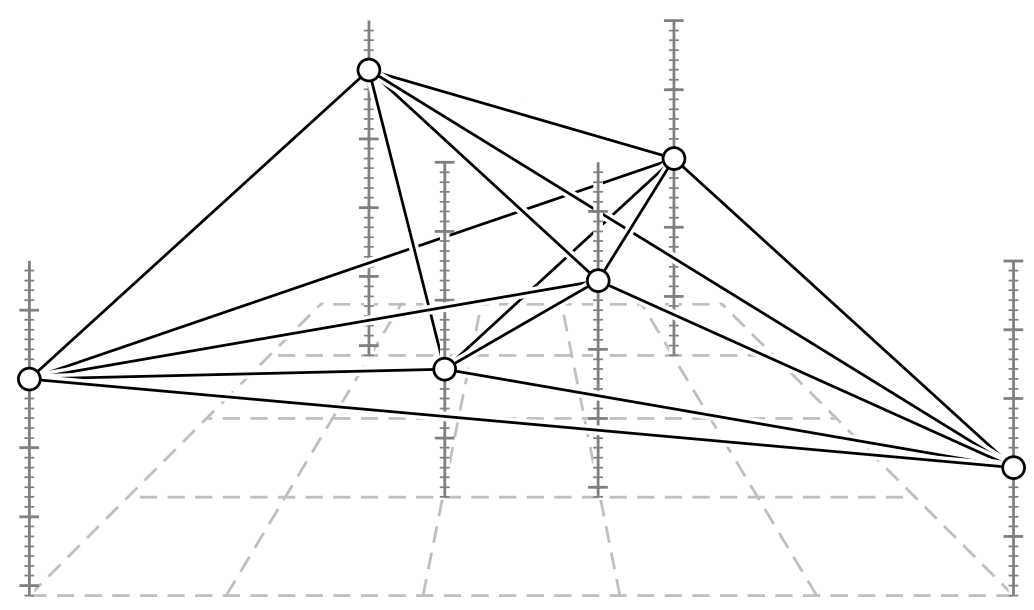

Fig. 1. A three-dimensional drawing of $K_{6}$.

Our algorithm for constructing an ordered layering makes use of the socalled normalised path-decompositions of Gupta et al. [20]. (The more general notion of normalised tree-decompositions was developed earlier by Gupta and Nishimura [19].) A path-decomposition $T_{1}, T_{2}, \ldots, T_{m}$ of width $k$ is normalised if $\left|T_{i}\right|=k+1$ for all odd $i$ and $\left|T_{i}\right|=k$ for all even $i$, and $T_{i-1} \cap T_{i+1}=T_{i}$ for all even $i$. The algorithm of Gupta et al. [20] normalises a path-decomposition while maintaining the width in linear time.

Lemma 2. If a graph $G$ has a normalised path-decomposition $T_{1}, T_{2}, \ldots, T_{m}$ of width $k-1$, then $G$ has an ordered $k$-layering with no X-crossing (see Fig. 2).

Proof. For every vertex $v \in V(G)$, let $T_{\alpha(v)}$ and $T_{\beta(v)}$ be the first and last bags containing $v$. Construct an ordered $k$-layering of $G$ as follows. Let $T_{1}=$ $\left\{v_{1}, v_{2}, \ldots, v_{k}\right\}$, and position each $v_{i}$ as the leftmost vertex on layer $i, 1 \leq i \leq k$. Since the path-decomposition is normalised, for all bags $T_{j}$ with $j$ even, there is a unique vertex $x_{j} \in T_{j-1} \backslash T_{j}$; that is, $\beta\left(x_{j}\right)=j-1$. Similarly, for all bags $T_{j}$ with $j>1$ odd, there is a unique vertex $y_{j} \in T_{j} \backslash T_{j-1}$; that is, $\alpha\left(y_{j}\right)=j$.

The remainder of the ordered layering is constructed by sweeping through the bags of the path-decomposition as follows. For all odd $j=3,5, \ldots, m$, position $y_{j}$ in the same layer as the vertex $x_{j-1}$ and immediately to the right of $x_{j-1}$. 


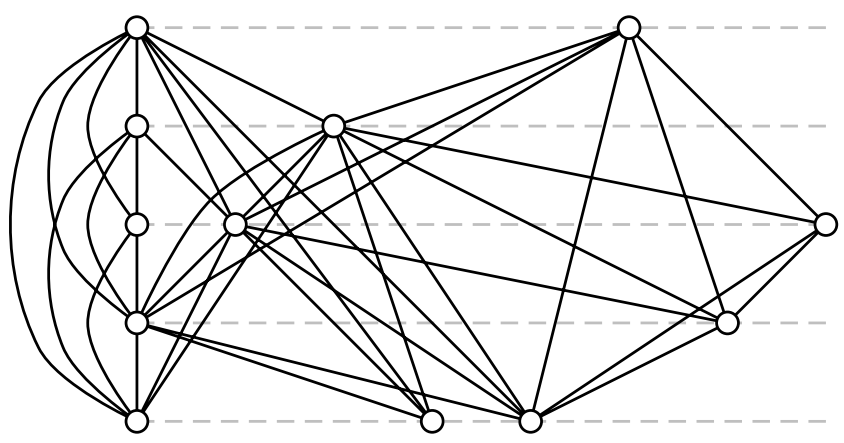

Fig. 2. An ordered 5-layering with no X-crossing produced by Lemma 2.

Clearly, $x_{j-1}$ was the rightmost vertex in the layer before inserting $y_{j}$. Since $j-1=\beta\left(x_{j-1}\right)<\alpha\left(y_{j}\right)=j$, there is no bag containing both $x_{j-1}$ and $y_{j}$, and no edge $x_{j-1} y_{j} \in E(G)$. In general, two vertices in the same layer are not in a common bag and are not adjacent.

Suppose there is an $\mathrm{X}$-crossing between edges $v w$ and $x y$. Without loss of generality, $v<_{i} x$ and $y<_{j} w$ for some layers $i$ and $j$. Thus $\beta(v)<\alpha(x)$ and $\beta(y)<\alpha(w)$. Since $v w$ is an edge, $v$ and $w$ appear in some bag together; that is, $\alpha(w) \leq \beta(v)$, which implies that $\beta(y)<\alpha(x)$. This is the desired contradiction since $x$ and $y$ appear in some bag together.

The second step of our algorithm is based on the algorithm of Pach et al. [30] for balancing the size of the colour classes in a vertex-colouring. Note that while Lemma 2 produces an ordered layering with no intra-layer edges, the remaining steps of our algorithm are valid in the more general situation that the given ordered layering possibly has intra-layer edges.

Lemma 3. If a graph $G$ has an ordered $k$-layering with no $X$-crossing, then for every $l>0, G$ has an ordered $\lfloor l+k\rfloor$-layering with no $X$-crossing and at most $\left\lceil\frac{n}{l}\right\rceil$ vertices in each layer.

Proof. For each layer with $q>\left\lceil\frac{n}{l}\right\rceil$ vertices, replace it by $\left\lceil q /\left\lceil\frac{n}{l}\right\rceil\right\rceil$ 'sub-layers' each with exactly $\left\lceil\frac{n}{l}\right\rceil$ vertices except for at most one sub-layer with $q \bmod \left\lceil\frac{n}{l}\right\rceil$ vertices, such that the vertices in each sub-layer are consecutive in the original layer and the original order is maintained. There is no X-crossing between sublayers of the same original layer as there is at most one edge between such sublayers. There is no X-crossing between sub-layers from different original layers as otherwise there would be an X-crossing in the original layering. There are at most $\lfloor l\rfloor$ layers with $\left\lceil\frac{n}{l}\right\rceil$ vertices. Since there are at most $k$ layers with less than $\left\lceil\frac{n}{l}\right\rceil$ vertices, one for each of the original layers, there is a total of at most $\lfloor l+k\rfloor$ layers.

The third step of our algorithm is inspired by the generalisations of the moment curve algorithm by Cohen et al. [8] and Pach et al. [30]. Loosely speaking, 
$k$ then chordal, interval and circular arc graphs also have bounded tree-width. Thus Corollary 1(b) pertains to such graphs.

Since a planar graph is 4-colourable, by the results of Calamoneri and Sterbini [5] and Pach et al. [30] discussed above, every planar graph has a threedimensional drawing with $O\left(n^{2}\right)$ volume. Of course this result also follows from the classical algorithms of de Fraysseix et al. [10] and Schnyder [33] for producing plane grid drawings. All of these methods produce $O(1) \times O(n) \times O(n)$ drawings, which have $\Theta(n)$ aspect ratio. Since every planar graph $G$ has $\operatorname{pw}(G) \in O(\sqrt{n})$ [2] we have the following corollary of Theorem 1.

Corollary 2. Every n-vertex planar graph has an $O(\sqrt{n}) \times O(\sqrt{n}) \times O(n)$ threedimensional drawing with $\Theta(\sqrt{n})$ aspect ratio.

This result matches the above $O\left(n^{2}\right)$ volume bounds with an improvement in the aspect ratio by a factor of $\Theta(\sqrt{n})$. Our final result examines the trade-off between aspect ratio and volume.

Theorem 2. Let $G$ be an $n$-vertex graph. For every $r, 1 \leq r \leq n /(\operatorname{pw}(G)+1)$, $G$ has a three-dimensional drawing with $O\left(n^{3} / r^{2}\right)$ volume and aspect ratio $2 r$.

\section{Proofs}

We first introduce a combinatorial structure which is the basis for a twodimensional layered graph drawing. An ordered $k$-layering of a graph $G$ consists of a partition $V_{1}, V_{2}, \ldots, V_{k}$ of $V(G)$ into layers, and a total ordering $<_{i}$ of each $V_{i}$, such that for every edge $v w$, if $v<_{i} w$ then there is no vertex $x$ with $v<_{i} x<_{i} w$. The span of an edge $v w$ is $|i-j|$ if $v \in V_{i}$ and $w \in V_{j}$. An intra-layer edge is an edge with zero span. An $X$-crossing consists of two edges $v w$ and $x y$ such that for distinct layers $i$ and $j, v<_{i} x$ and $y<_{j} w$. The next lemma highlights the intrinsic relationship between three-dimensional drawings and ordered layerings.

Lemma 1. Let $G$ be an n-vertex graph with an $A \times B \times C$ three-dimensional drawing. Then $G$ has an ordered $A B$-layering with no $X$-crossing, and $G$ has an ordered $2 A B$-layering with no $X$-crossing and no intra-layer edges.

Proof. Let $V_{x, y}$ be the set of vertices of $G$ with an $X$-coordinate of $x$ and a $Y$-coordinate of $y$, where without loss of generality $1 \leq x \leq A$ and $1 \leq y \leq Y$. Consider each set $V_{x, y}$ to be ordered $V_{x, y}=\left(v_{x, y, 1}, \ldots, v_{x, y, n_{x, y}}\right)$ by the $Z$ coordinates of its elements. Then the ordered layering $\left\{V_{x, y}: 1 \leq x \leq A, 1 \leq\right.$ $y \leq Y\}$ has no X-crossing as otherwise there would be a crossing in the original drawing. Now, define $V_{x, y}^{\prime}=\left\{v_{x, y, j}: j\right.$ odd $\}$ and $V_{x, y}^{\prime \prime}=\left\{v_{x, y, j}: j\right.$ even $\}$, and consider these sets to be ordered as in $V_{x, y}$. Then, as in the above, the ordered layering $\left\{V_{x, y}^{\prime}, V_{x, y}^{\prime \prime}: 1 \leq x \leq A, 1 \leq y \leq B\right\}$ has no X-crossing. Moreover there is no intra-layer edges, as otherwise an edge between two vertices in $V_{x, y}^{\prime}$ would have passed through a vertex in $V_{x, y}^{\prime \prime}$ (or vice versa) in the original drawing. 
Cohen et al. [8] allow three 'free' dimensions, whereas Pach et al. [30] use the assignment of vertices to colour classes to 'fix' one dimension with two dimensions free. We use an assignment of vertices to layers in an ordered layering without $\mathrm{X}$-crossings to fix two dimensions with one dimension free.

Lemma 4. If a graph $G$ has an ordered $k$-layering $\left\{\left(V_{i},<_{i}\right): 1 \leq i \leq k\right\}$ with no $X$-crossing then $G$ has a $k \times 2 k \times 2 k \cdot n^{\prime}$ three-dimensional drawing, where $n^{\prime}$ is the maximum number of vertices in a layer.

Proof. Let $p$ be the smallest prime such that $p>k$. Then $p \leq 2 k$ by Bertrand's postulate. For each $i, 1 \leq i \leq k$, represent the vertices in $V_{i}$ by the grid-points

$$
\left\{\left(i, i^{2} \bmod p, t\right): 1 \leq t \leq p \cdot\left|V_{i}\right|, t \equiv i^{3}(\bmod p)\right\},
$$

such that the $Z$-coordinates respect the given linear ordering $<_{i}$. Draw each edge as a line-segment between its end-vertices. Suppose two edges $e$ and $e^{\prime}$ cross such that their end-vertices are at distinct points $\left(i_{\alpha}, i_{\alpha}^{2} \bmod p, t_{\alpha}\right), 1 \leq \alpha \leq 4$. Then these points are coplanar, and if $M$ is the matrix

$$
M=\left(\begin{array}{cccc}
1 & i_{1} & i_{1}^{2} \bmod p & t_{1} \\
1 & i_{2} & i_{2}^{2} \bmod p & t_{2} \\
1 & i_{3} & i_{3}^{2} \bmod p & t_{3} \\
1 & i_{4} & i_{4}^{2} \bmod p & t_{4}
\end{array}\right)
$$

then the determinant $\operatorname{det}(M)=0$. We proceed by considering the number of distinct layers $N=\left|\left\{i_{1}, i_{2}, i_{3}, i_{4}\right\}\right|$.

- $N=1$ : By the definition of an ordered layering $e$ and $e^{\prime}$ do not cross.

- $N=2$ : If either edge is intra-layer then $e$ and $e^{\prime}$ do not cross. Otherwise neither edge is intra-layer, and since there are no X-crossings in the ordered layering, $e$ and $e^{\prime}$ do not cross.

- $N=3$ : Without loss of generality $i_{1}=i_{2}$. It follows that $\operatorname{det}(M)=$ $\left(t_{2}-t_{1}\right) \cdot \operatorname{det}\left(M^{\prime}\right)$, where

$$
M^{\prime}=\left(\begin{array}{ccc}
1 & i_{2} & i_{2}^{2} \bmod p \\
1 & i_{3} & i_{3}^{2} \bmod p \\
1 & i_{4} & i_{4}^{2} \bmod p
\end{array}\right)
$$

Since $t_{1} \neq t_{2}, \operatorname{det}\left(M^{\prime}\right)=0$. However, $M^{\prime}$ is a Vandermonde matrix modulo $p$, and thus

$$
\operatorname{det}\left(M^{\prime}\right) \equiv\left(i_{2}-i_{3}\right)\left(i_{2}-i_{4}\right)\left(i_{3}-i_{4}\right) \quad(\bmod p),
$$

which is non-zero since $i_{2}, i_{3}$ and $i_{4}$ are distinct and $p$ is a prime, a contradiction.

- $N=4$ : Let $M^{\prime}$ be the matrix obtained from $M$ by taking each entry modulo $p$. Then $\operatorname{det}\left(M^{\prime}\right)=0$. Since $t_{\alpha} \equiv i_{\alpha}^{3}(\bmod p), 1 \leq \alpha \leq 4$,

$$
M^{\prime} \equiv\left(\begin{array}{cccc}
1 & i_{1} & i_{1}^{2} & i_{1}^{3} \\
1 & i_{2} & i_{2}^{2} & i_{2}^{3} \\
1 & i_{3} & i_{3}^{2} & i_{3}^{3} \\
1 & i_{4} & i_{4}^{2} & i_{4}^{3}
\end{array}\right) \quad(\bmod p)
$$


Since each $i_{\alpha}<p, M^{\prime}$ is a Vandermonde matrix modulo $p$, and thus

$$
\operatorname{det}\left(M^{\prime}\right) \equiv\left(i_{1}-i_{2}\right)\left(i_{1}-i_{3}\right)\left(i_{1}-i_{4}\right)\left(i_{2}-i_{3}\right)\left(i_{2}-i_{4}\right)\left(i_{3}-i_{4}\right) \quad(\bmod p),
$$

which is non-zero since $i_{\alpha} \neq i_{\beta}$ and $p$ is a prime. This contradiction proves there are no edge crossings. The produced drawing is at most $k \times 2 k \times 2 k \cdot n^{\prime}$.

We now prove the theorems.

Proof of Theorem 1. By Lemma 2, $G$ has an ordered $k$-layering with no Xcrossing, where $k=\operatorname{pw}(G)+1$. By Lemma 3 with $l=k, G$ has an ordered $(2 k)$-layering with no $\mathrm{X}$-crossing and at most $\left\lceil\frac{n}{k}\right\rceil$ vertices on each layer. By Lemma $4, G$ has a $2 k \times 4 k \times 4 k \cdot\left\lceil\frac{n}{k}\right\rceil$ three-dimensional drawing, which is at most $2(\operatorname{pw}(G)+1) \times 4(\operatorname{pw}(G)+1) \times 4(n+\operatorname{pw}(G)+1)$. The result follows since $1 \leq \operatorname{pw}(G)<n$.

Proof of Theorem 2. By Lemma 2, $G$ has an ordered $k$-layering with no Xcrossing, where $k=\operatorname{pw}(G)+1$. By Lemma 3 with $l=\frac{n}{r}, G$ has an ordered $\left\lfloor\frac{n}{r}+k\right\rfloor$-layering with no $\mathrm{X}$-crossing and at most $r$ vertices in each layer. By assumption $r \leq n /(\operatorname{pw}(G)+1)$. Thus $k \leq \frac{n}{r}$ and the number of layers is at most $\frac{2 n}{r}$. By Lemma $4, G$ has a $\frac{2 n}{r} \times \frac{4 n}{r} \times 4 n$ three-dimensional drawing, which has volume $32 n^{3} / r^{2}$ and aspect ratio $2 r$.

\section{Commentary}

Consider the following open problems concerning straight-line grid drawings.

1. A graph with degree bounded by some constant $k$ is $(k+1)$-colourable, and thus by the theorem of Pach et al. [30], has a three-dimensional drawing with $O\left(n^{2}\right)$ volume. Pach et al. [30] ask whether every graph with bounded degree has a three-dimensional drawing with $o\left(n^{2}\right)$ volume?

2. As discussed in Section 1 every planar graph has a three-dimensional drawing with $O\left(n^{2}\right)$ volume. Felsner et al. [17] ask whether every planar graph has a three-dimensional drawing with $O(n)$ volume? Even a volume bound of $o\left(n^{2}\right)$ would be interesting.

As a final observation, we show that a generalisation of the 'wrapping' algorithm of Felsner et al. [17] can be applied in conjunction with our algorithm, which may be helpful in solving the above open. Note that Felsner et al. [17] prove the case $s=1$ (with improved constants in the volume).

Lemma 5. Let a graph $G$ have an ordered $k$-layering $\left\{\left(V_{i},<_{i}\right): 1 \leq i \leq k\right\}$ with no $X$-crossing. If the maximum edge span is $s$, then $G$ has an $O(s) \times O(s) \times O(n)$ three-dimensional drawing.

Proof. Let $t=2 s+1$. Construct an ordered $t$-layering of $G$ by merging the layers $\left\{V_{i}: i \equiv j(\bmod t)\right\}$ for each $j, 0 \leq j \leq t-1$, with vertices in $V_{\alpha}$ appearing before vertices in $V_{\beta}$ in the new layer $j$, for all $\alpha, \beta \equiv j(\bmod t)$ with 
$\alpha<\beta$. The given ordering of each $V_{i}$ is preserved in the new layers. It remains to prove that there is no $\mathrm{X}$-crossing. Consider two edges $v w$ and $x y$. Let $i_{1}$ and $i_{2}, 1 \leq i_{1}<i_{2} \leq k$, be the minimum and maximum layers containing $v, w, x$ or $y$ in the ordered $k$-layering.

Firstly consider the case that $i_{2}-i_{1}>2 s$. Then without loss of generality $v$ is on layer $i_{2}$ and $y$ is on layer $i_{1}$. Thus $w$ is on a greater layer than $x$, and even if $x$ (or $y$ ) appear on the same layer as $v$ (or $w$ ) in the new $t$-layering, $x$ (or $y$ ) will be to the left of $v$ (or $w$ ). Thus these edges do not form an X-crossing in the ordered $t$-layering. Otherwise $i_{2}-i_{1} \leq 2 s$. Thus any two of $v, w, x$ or $y$ will appear on the same layer in the $t$-layering if and only if they are on the same layer in the given ordered $k$-layering ( four vertices to appear on exactly two layers in the ordered $t$-layering is if they were on exactly two layers in the given $k$-layering, in which case, by assumption $v w$ and $x y$ do not form an X-crossing.

Therefore there are no X-crossings. By Lemma 3 with $l=t, G$ has an ordered $2 t$-layering with no X-crossing and at most $\left\lceil\frac{n}{t}\right\rceil$ vertices in each layer. Since $t=2 s+1$, by Lemma $4, G$ has a $2(2 s+1) \times 4(2 s+1) \times 4(2 s+1)\left\lceil\frac{n}{2 s+1}\right\rceil$ threedimensional drawing, which is $2(2 s+1) \times 4(2 s+1) \times 4(n+2 s)$. The result follows since $s \leq n$.

Lemma 5 also shows that small path-width is not necessary for a graph to have a three-dimensional drawing with small volume. The $\sqrt{n} \times \sqrt{n}$ plane grid graph has path-width $\Theta(\sqrt{n})$, but has an ordered layering with maximum edge span 1 . Therefore it has a three-dimensional drawing with $O(n)$ volume by Lemma 5 .

\section{Note Added in Proof}

The results in this paper have recently been extended. In particular, Wood [38] has proved that every graph $G$ from a proper minor-closed family has a $O(1) \times$ $O(1) \times O(n)$ three-dimensional drawing if and only if $G$ has $O(1)$ queue-number, and Dujmović and Wood [14] have proved that graphs of bounded tree-width have three-dimensional drawings with $O(n)$ volume.

\section{Acknowledgements}

The authors are grateful for stimulating discussions with Prosenjit Bose, Hubert de Fraysseix, Stefan Langerman, Giuseppe Liotta, and Patrice Ossona de Mendez.

\section{References}

[1] H. L. Bodlaender, A linear-time algorithm for finding treedecompositions of small treewidth. SIAM J. Comput., 25(6):1305-1317, 1996. 
[2] H. L. Bodlaender, A partial $k$-arboretum of graphs with bounded treewidth. Theoret. Comput. Sci., 209(1-2):1-45, 1998.

[3] F. J. Brandenburg, ed., Proc. International Symp. on Graph Drawing (GD '95), vol. 1027 of Lecture Notes in Comput. Sci., Springer, 1996.

[4] I. BRUß AND A. FrICK, Fast interactive 3-D graph visualization. In [3], pp. 99-110.

[5] T. Calamoneri and A. Sterbini, 3D straight-line grid drawing of 4colorable graphs. Inform. Process. Lett., 63(2):97-102, 1997.

[6] K. Chilakamarri, N. Dean, and M. Littman, Three-dimensional Tutte embedding. In Proc. 26th Southeastern International Conf. on Combinatorics, Graph Theory and Computing, vol. 107 of Cong. Numer., pp. 129140, 1995.

[7] M. Chrobak, M. Goodrich, and R. Tamassia, Convex drawings of graphs in two and three dimensions. In Proc. 12th Annual ACM Symp. on Comput. Geom., pp. 319-328, 1996.

[8] R. F. Cohen, P. Eades, T. Lin, and F. Ruskey, Three-dimensional graph drawing. Algorithmica, 17(2):199-208, 1996.

[9] I. F. Cruz AND J. P. TwaroG, 3D graph drawing with simulated annealing. In [3], pp. 162-165.

[10] H. de Fraysseix, J. Pach, And R. Pollack, How to draw a planar graph on a grid. Combinatorica, 10(1):41-51, 1990.

[11] E. di Giacomo, G. Liotta, and S. Wismath, Drawing series-parallel graphs on a box. In S. Wismath, ed., Proc. 14th Canadian Conf. on Computational Geometry (CCCG '02), The University of Lethbridge, Canada, 2002.

[12] R. G. Downey And M. R. Fellows, Parameterized complexity. Springer, 1999.

[13] V. Dujmović, M. Fellows, M. Hallett, M. Kitching, G. Liotta, C. McCartin, N. Nishimura, P. Ragde, F. Rosemand, M. SuderMAn, S. Whitesides, And D. R. Wood, On the parameterized complexity of layered graph drawing. In F. Meyer Auf Der HeIde, ed., Proc. 5th Annual European Symp. on Algorithms (ESA '01), vol. 2161 of Lecture Notes in Comput. Sci., pp. 488-499, Springer, 2001.

[14] V. Dujmović And D. R. Wood, Tree-partitions of $k$-trees with applications in graph layout. Tech. Rep. TR-02-03, School of Computer Science, Carleton University, Ottawa, Canada, 2002.

[15] P. Eades And P. Garvan, Drawing stressed planar graphs in three dimensions. In [3], pp. 212-223.

[16] P. Erdös, Appendix. In K. F. Roth, On a problem of Heilbronn. J. London Math. Soc., 26:198-204, 1951.

[17] S. Felsner, S. Wismath, and G. Liotta, Straight-line drawings on restricted integer grids in two and three dimensions. In [28], pp. 328-342.

[18] A. Garg, R. Tamassia, And P. VoccA, Drawing with colors. In J. Diaz And M. Serna, eds., Proc. 4th Annual European Symp. on Algorithms (ESA '96), vol. 1136 of Lecture Notes in Comput. Sci., pp. 12-26, Springer, 1996. 
[19] A. Gupta And N. Nishimura, Sequential and parallel algorithms for embedding problems on classes of partial $k$-trees. In Proc. 4th Scandinavian Workshop on Algorithm Theory (SWAT '94), vol. 824 of Lecture Notes in Comput. Sci., pp. 172-182, Springer, 1984.

[20] A. Gupta, N. Nishimura, A. Proskurowski, and P. Ragde, Embeddings of $k$-connected graphs of pathwidth $k$. In M. M. Halldorsson, ed., Proc. 7th Scandinavian Workshop on Algorithm Theory (SWAT '00), vol. 1851 of Lecture Notes in Comput. Sci., pp. 111-124, Springer, 2000.

[21] P. HLinĔNÝ, Crossing-critical graphs and path-width. In [28], pp. 102-114.

[22] S.-H. Hong, Drawing graphs symmetrically in three dimensions. In [28], pp. 189-204.

[23] S.-H. Hong And P. EAdes, An algorithm for finding three dimensional symmetry in series parallel digraphs. In D. LEE And S.-H. TEng, eds., Proc. 11th International Conf. on Algorithms and Computation (ISAAC '00), vol. 1969 of Lecture Notes in Comput. Sci., pp. 266-277, Springer, 2000.

[24] S.-H. Hong And P. EAdes, An algorithm for finding three dimensional symmetry in trees. In J. MARKs, ed., Proc. 8th International Symp. on Graph Drawing (GD '00), vol. 1984 of Lecture Notes in Comput. Sci., pp. 360-371, Springer, 2001.

[25] S.-H. Hong, P. Eades, A. Quigley, And S.-H. Lee, Drawing algorithms for series-parallel digraphs in two and three dimensions. In S. WHITESIDES, ed., Proc. 6th International Symp. on Graph Drawing (GD '98), vol. 1547 of Lecture Notes in Comput. Sci., pp. 198-209, Springer, 1998.

[26] F. T. Leighton And A. L. Rosenberg, Three-dimensional circuit layouts. SIAM J. Comput., 15(3):793-813, 1986.

[27] B. Monien, F. Ramme, And H. Salmen, A parallel simulated annealing algorithm for generating 3D layouts of undirected graphs. In [3], pp. 396408.

[28] P. Mutzel, M. Jünger, And S. Leipert, eds., Proc. 9th International Symp. on Graph Drawing (GD '01), vol. 2265 of Lecture Notes in Comput. Sci., Springer, 2002.

[29] D. I. Ostry, Some Three-Dimensional Graph Drawing Algorithms. Master's thesis, Department of Computer Science and Software Engineering, The University of Newcastle, Australia, 1996.

[30] J. Pach, T. Thiele, and G. Tóth, Three-dimensional grid drawings of graphs. In G. Di BAtTista, ed., Proc. 5th International Symp. on Graph Drawing (GD '97), vol. 1353 of Lecture Notes in Comput. Sci., pp. 47-51, Springer, 1998.

[31] Z. PEng, Drawing Graphs of Bounded Treewidth/Pathwidth. Master's thesis, Department of Computer Science, University of Auckland, New Zealand, 2001.

[32] T. Poranen, A new algorithm for drawing series-parallel digraphs in 3D. Tech. Rep. A-2000-16, Dept. of Computer and Information Sciences, University of Tampere, Finland, 2000. 
[33] W. Schnyder, Planar graphs and poset dimension. Order, 5(4):323-343, 1989.

[34] M. Thorup, All structured programs have small tree-width and good register allocation. Information and Computation, 142(2):159-181, 1998.

[35] C. WARE AND G. Franck, Viewing a graph in a virtual reality display is three times as good as a $2 \mathrm{D}$ diagram. In A. L. AMBler AND T. D. KimurA, eds., Proc. IEEE Symp. Visual Languages (VL '94), pp. 182-183, IEEE, 1994.

[36] C. Ware, D. Hui, And G. Franck, Visualizing object oriented software in three dimensions. In Proc. IBM Centre for Advanced Studies Conf. (CASCON '93), pp. 1-11, 1993.

[37] R. Weiskircher, Drawing planar graphs. In M. Kaufmann AND D. WAGner, eds., Drawing Graphs: Methods and Models, vol. 2025 of Lecture Notes in Comput. Sci., pp. 23-45, Springer, 2001.

[38] D. R. Wood, Queue layouts, tree-width, and three-dimensional graph drawing. Tech. Rep. TR-02-02 (revised), School of Computer Science, Carleton University, Ottawa, Canada, August, 2002. 